\title{
Importance of Hierarchical Structures in Wetting Stability on Submersed Superhydrophobic Surfaces
}

\author{
Yahui Xue, ${ }^{\dagger}$ Shigan $\mathrm{Chu},{ }^{\dagger}$ Pengyu Lv, ${ }^{\dagger}$ and Huiling Duan ${ }^{*}, \dagger, \S$ \\ ${ }^{\dagger}$ State Key Laboratory for Turbulence and Complex System, Department of Mechanics and Aerospace Engineering, College of \\ Engineering, Peking University, Beijing 100871, China \\ ${ }^{\ddagger}$ Key Laboratory of High Energy Density Physics Simulation (HEDPS), CAPT, Peking University, Beijing 100871, China \\ ${ }^{\S}$ State Key Laboratory of Nonlinear Mechanics (LNM), Institute of Mechanics, Chinese Academy of Sciences, Beijing 100190, China
}

ABSTRACT: Submersed superhydrophobic surfaces exhibit great potential for reducing flow resistance in microchannels and drag of submersed bodies. However, the low stability of liquid-air interfaces on those surfaces limits the scope of their application, especially under high liquid pressure. In this paper, we first investigate the wetting states on submersed hydrophobic surfaces with one-level structure under hydrostatic pressure. Different equilibrium states based on free-energy minimization are formulated, and their stabilities are analyzed as well. Then, by comparison with the existing numerical and experimental studies, we confirm that a new metastable state, which happens after depinning of the three-phase contact line
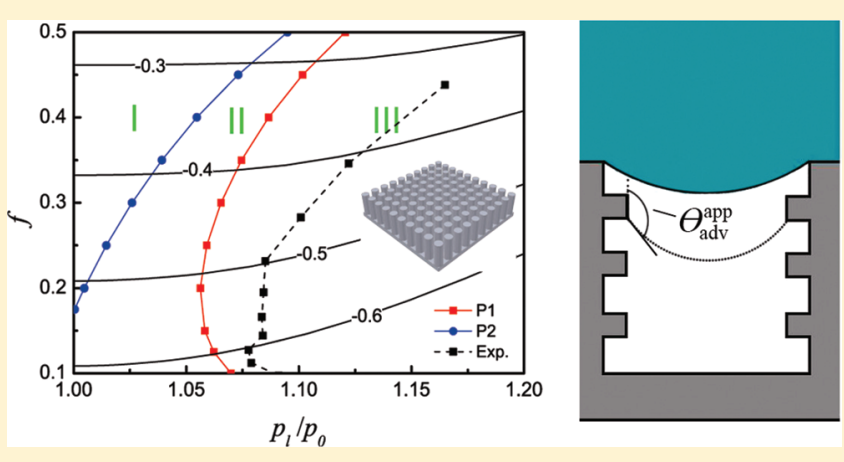

(TCL), exists. Finally, we show that a strategy of using hierarchical structures can strengthen the TCL pinning of the liquid-air interface in the metastable state. Therefore, the hierarchical structure on submersed surfaces is important to further improve the stability of superhydrophobicity under high liquid pressure.

\section{INTRODUCTION}

Wetting on rough surfaces has been paid much attention since the pioneer works of Wenzel $^{1}$ and Cassie and Baxter. ${ }^{2}$ Liquid can penetrate the valleys of the surface roughness or suspends over the peaks with air trapped underneath. These two regimes are called the Wenzel and Cassie-Baxter (CB) states, respectively. It is known that superhydrophobic surfaces exhibiting contact angles (CAs) greater than $150^{\circ}$ with little CA hysteresis (i.e., the difference between the advancing and receding CAs) can be obtained by an optimized combination of surface roughness and hydrophobicity in the $\mathrm{CB}$ state. ${ }^{3}$ However, various factors, including pressurization, ${ }^{4}$ vibration, ${ }^{5}$ and droplet impact, ${ }^{6,7}$ can induce the wetting transition from a $\mathrm{CB}$ to Wenzel state, and make the surface lose its superhydrophobicity.

Recent research interest arises in studying the behavior of submersed superhydrophobic surfaces due to the emergent requirement for drag reduction in microfluidic devices or submersed bodies. ${ }^{8,9}$ When the superhydrophobic surface is fully submersed in water, a closed-air film will be formed, which differs from the state of a droplet placed on an open surface structure. In the former case, the air pressure is dependent on the pressurization by the liquid; while in the latter, the air pressure is usually assumed to be constant and equal to the ambient one. For the submersed superhydrophobic surfaces, the existence of trapped air is essential for a large slip length, and this large slip length can be obtained by increasing the area fraction of the liquid-air interface. ${ }^{8}$ However, the diffusion of the trapped air into the liquid or high liquid pressure will induce the collapse of the liquid-air interface, and finally induce the failure of the superhydrophobic state. Therefore, to keep the superhydrophobic state of submersed surfaces, the stability of the liquid-air interface under hydrostatic pressure is essential.

Several experiments have been carried out to study the wetting transition of the submersed superhydrophobic surfaces by using optical techniques. ${ }^{10-13}$ Lei et al. ${ }^{10}$ studied the behavior of a superhydrophobic surface made of a PDMS (polydimethylsiloxane) grating by using the transmission diffraction technique, and it was found that the transitions can be identified by observing an irreversible change of the diffraction pattern. Rathgen et al. ${ }^{11}$ proposed an optical diffraction technique by using the reflection manner to study the microscopic shape, contact angle, and Laplace law behavior of the liquid-gas interfaces at a submersed superhydrophobic surface. Poetes et al. ${ }^{12}$ used the method of light reflection to study the decay of the plastron on the submersed rough surfaces. Forsberg et al. ${ }^{13}$ applied a similar method as Poetes et al. ${ }^{12}$ to study the wetting transitions on superhydrophobic surfaces under hydrostatic pressure, and they first demonstrated that a closed film of trapped air helps stabilize the submersed $\mathrm{CB}$ state, especially in the low pillar densities. Moreover, based

Received: January 22, 2012

Revised: April 15, 2012

Published: May 29, 2012 
on the numerical method developed by Lobaton and Salamon, ${ }^{14}$ Forsberg et al. ${ }^{13}$ calculated the constant mean curvature of the meniscus between pillars by considering the increasing air pressure as the meniscus curvature increases. It is noted that Forsberg et al. ${ }^{13}$ supposed that the meniscus collapse would happen when the whole three-phase contact line (TCL) depins from the pillar tops. However, there exists a significant deviation between the predicted collapse pressure and that obtained by their experiments, and the deviation is especially high as the increase of the pillar densities.

On the other hand, inspired by the unique water-repellent capabilities of some plant leaves and insect legs, researchers have studied the special hierarchical structures of these plant leaves and insect legs and have shown that the nanomicrohierarchical structure is crucial for their stability. ${ }^{15-17}$ The nanostructure helps sustain a high liquid pressure and the microstructure reduces the contact area. The synergy between the micro- and nanoroughness stabilizes the superhydrphobic state by enlarging the energy barrier between the $\mathrm{CB}$ and Wenzel states. ${ }^{17}$ Moreover, it is shown that the hierarchical structures can increase the wetting stability of the submersed superhydrophobic surfaces. For example, Lee et al. ${ }^{18}$ investigated the influence of hierarchical structures of superhydrophobic surfaces on liquid slip by experiments. In their experiment, Lee et al. ${ }^{18}$ achieved such hierarchical structure with the smooth tops and nanostructured sidewalls (as illustrated in Figure 1), which enhanced the stability of the

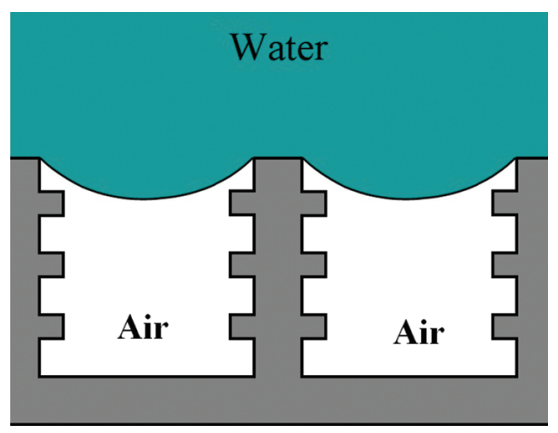

Figure 1. Schematic of a two-level hierarchical superhydrophobic surface with smooth tops and nanostructured sidewalls. ${ }^{18}$

liquid-air interfaces at a high air fraction $(\sim 100 \%)$ and extremely increased the slip length as well. Though the experiments have shown that the stability of submersed superhydrohphobic surfaces can be improved by hierarchical structures with nanostructured sidewalls (c.f., Figure 1), there is a lack of quantitative explanation and understanding.

In this paper, we establish a theoretical formulation to investigate the wetting stability on the submersed superhydrophobic surfaces under hydrostatic pressure. Special attention is paid to the transition process from a $\mathrm{CB}$ to Wenzel state. Our formulation is based on the structured surface with cylindrical pores on a square lattice. The equilibrium states of wetting on such surfaces are predicted, and their stabilities are analyzed comprehensively. Then, the above formulation is generalized to explain the behavior of a pillared surface, which has been observed in experiments. A comparison of our theoretical results with those from the available experiments is given. Finally, based on the above formulation and analysis, we quantitatively show the importance of hierarchical structures with structured sidewalls in wetting stability of submersed superhydrophobic surfaces under hydrostatic pressure.

\section{FORMULATION}

From the energy point of view, the wetting transition can be regarded as a process of overcoming the energy barrier between the CB and Wenzel states. ${ }^{19}$ Energy minimization theory has been widely used to interpret the wetting transition of droplets on the superhydrophobic surfaces. ${ }^{19-22}$ Note that the capillary length is defined by $a=(\gamma / \rho g)^{1 / 2}$, where $\gamma$ is the liquid surface tension, $\rho$ the liquid density, and $g$ the gravity acceleration. When the droplet size is much smaller than $a$, the effect of gravity is usually negligible. The energy minimization is generally based on calculating the total surface energy for an isothermal and closed system, e.g., a droplet settling on an open microstructure surface. ${ }^{20,21}$ For a rough surface with cavities, the compressibility of the trapped air in cavities will influence the wetting states of the droplet and should be taken into account. ${ }^{22,23}$ When a superhydrophobic surface is submersed in water, the hydrostatic pressure, usually comparable to or even higher than the capillary pressure, will play an important role in the wetting transitions. ${ }^{13}$ Besides, a closed-air film will be formed and the protruding meniscus will increase the air pressure. Patankar ${ }^{22}$ calculated the free energy differences in different wetting states of a droplet on a cavity-patterned substrate, with respect to the $\mathrm{CB}$ state as a reference one.

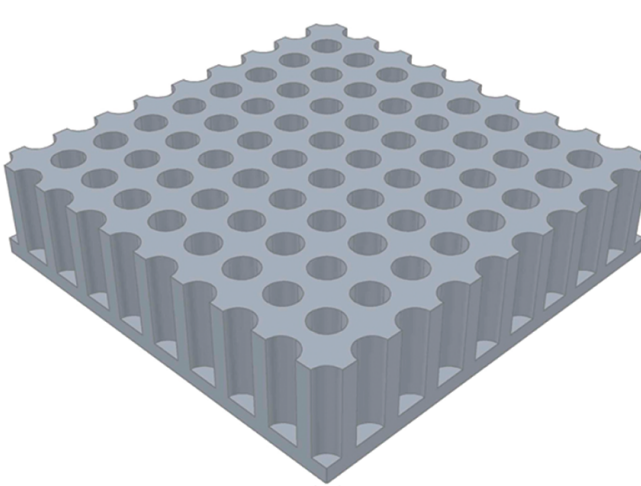

(a)

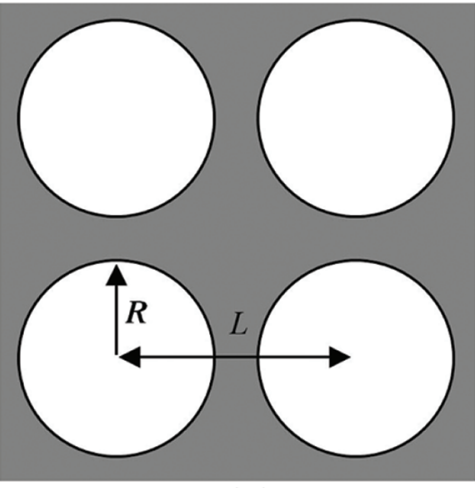

(b)

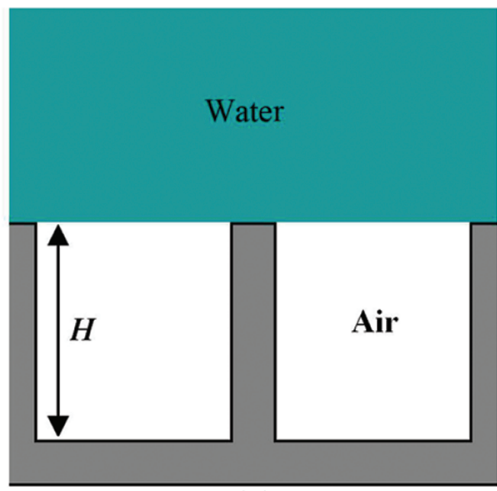

(c)

Figure 2. Schematic of microstructured surfaces with cylindrical pores of radius $R$ and depth $H$ on a periodic square lattice of constant $L$. (a) 3D view; (b) top view; (c) side view with water on top (i.e., the classical CB state). 

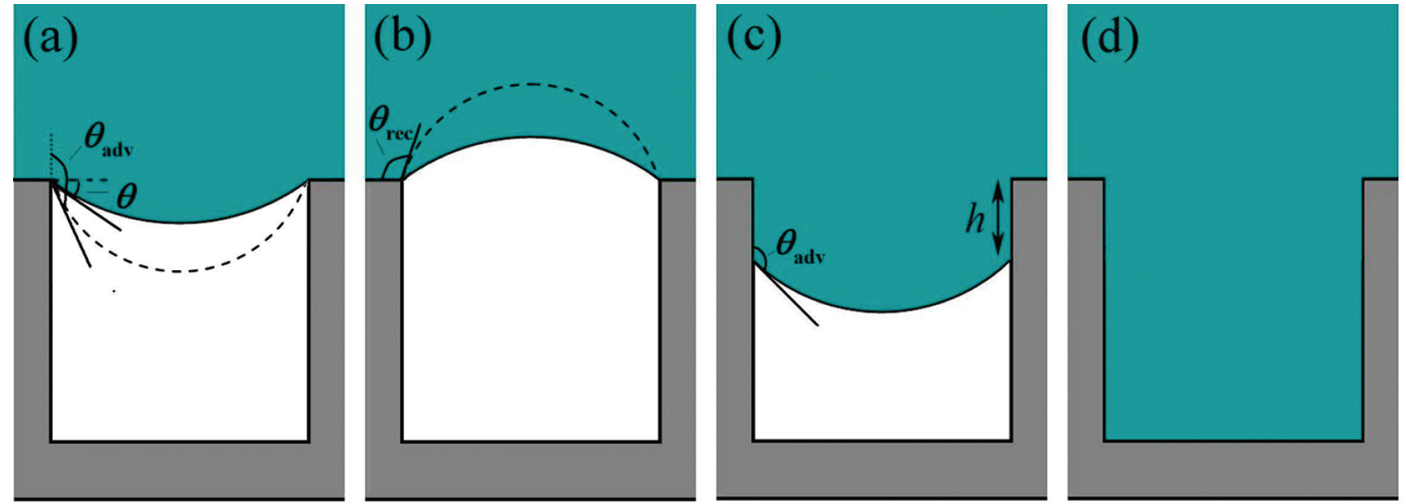

Figure 3. Schematic illustrating the morphologies of the liquid-air interface: (a-b) during pinning; (c) after depinning; (d) the Wenzel state. The dashed lines in (a) and (b) show the critical positions of the advancing and receding angles $\theta_{\text {adv }}$ and $\theta_{\text {rec }}$ respectively, where the depinning happens.

In the following, we will give a formulation to predict the wetting states of submersed surperhydrophobic surfaces under hydrostatic pressure, based on minimizing the total free energy of the system. In our analysis, the structure size under consideration is much smaller than the capillary length $a$ so that a meniscus with constant curvature can be assumed. Moreover, the depth of cavities is large enough such that the meniscus will not touch the substrate, and will not induce the wetting transition during the process under consideration. In order to capture the main physical picture, for brevity, we first set up a formulation in terms of a relatively simple structure, i.e., cylindrical pores on a periodic square lattice as shown in Figure 2. Then, this formulation based on pore structures will be extended to investigate pillared and hierarchical structures in the later sections.

The surface structure is submersed in an incompressible liquid (e.g., water), and a hydrophobic solid material is considered. The whole system is assumed to be an isothermal ensemble. The ambient air is at constant temperature $T_{0}$ and pressure $p_{0}$, and air diffusion into and out of the liquid is neglected. Then, the free energy $G$, which governs the equilibrium and stability of the system, is expressed as ${ }^{22,24}$

$$
G=G_{\mathrm{b}}+G_{\mathrm{g}}+G_{\mathrm{s}}
$$

where $G_{\mathrm{b}}$ is the bulk energy, $G_{\mathrm{g}}$ is the potential energy due to body forces (e.g., gravity), and $G_{s}$ is the total surface energy.

We choose the classical $\mathrm{CB}$ state (c.f., Figure $2 \mathrm{~b}$ ) as the reference state, in which the liquid-air interface is planar and the air trapped in each pore is at atmospheric pressure $p_{0}$ and volume $V_{0}\left(=\pi R^{2} H\right) . R$ and $H$ are the pore radius and depth, respectively. The nearest distance between the pores is $L$. The liquid protrudes into the pores and pressurizes the trapped air inside, which gives rise to the bulk energy $\left(G_{b}\right)$ of the $\operatorname{air}^{22}$

$$
\begin{aligned}
G_{b} & =I \cdot\left[\left(p_{0}-p_{1}\right) V_{1}+\int_{p_{0}}^{p_{1}} V \mathrm{~d} p\right] \\
& =I \cdot\left[\left(p_{0}-p_{1}\right) V_{1}+n k T \ln \frac{p_{1}}{p_{0}}\right]
\end{aligned}
$$

where the ideal gas law $p V=n k T$ is assumed, $n$ is the number of moles of gas, $k$ is the gas constant, and $V, p$, and $T$ represent volume, pressure, and temperature, respectively. The subscripts 0 and 1 denote the reference and final states of the air, respectively. $I$ is the number of the pores submersed under the liquid. The volume of the liquid protruding into each pore on average is expressed as

$$
V_{\text {in }}=\frac{\pi R^{3}}{3 \sin ^{3} \theta}\left(2-3 \cos \theta+\cos ^{3} \theta\right)+\pi R^{2} h
$$

where a spherical configuration of the liquid-air interface is assumed, $h$ is the liquid protrusion depth, and $\theta$ is the sagging angle of the liquid-air interface (which is defined in Figure 3a). The meniscus can be concave $(\theta>0)$ or convex $(\theta<0)$, depending on the hydrostatic pressure applied. Then, the final volume of the air in each pore is

$$
V_{1}=\pi R^{2} \cdot H-V_{\text {in }}
$$

The change in the potential energy of the liquid (e.g., due to the reduction of the gravity potential) can be expressed as

$$
G_{g} \approx\left(p_{0}-p_{1}\right) \cdot V_{\text {in }} \cdot I
$$

where $p_{1}$ is the liquid pressure. The total surface energy is

$$
G_{\mathrm{s}}=\gamma_{\mathrm{lg}}\left[2 \pi R^{2} \frac{1-\cos \theta}{\sin ^{2} \theta}-\pi R^{2}-2 \pi R h \cos \theta_{\mathrm{e}}\right] \cdot I
$$

where $f$ is the solid fraction of the substrate and $\theta_{\mathrm{e}}$ is the equilibrium contact angle defined by the Young equation, i.e., $\cos \theta_{\mathrm{e}}=\left(\gamma_{\mathrm{sg}}-\gamma_{\mathrm{sl}}\right) / \gamma_{\mathrm{lg}}$. The subscripts $s, g$, and $l$ denote the solid, air, and liquid, respectively. Note that $n k T=p_{0} V_{0}$ and $V_{1}$ $=V_{0}-V_{\text {in }}$. Combining eqs $1-2$ and 5-6, we get the nondimensionalized total free energy $\tilde{G}\left(=G(1-f) /\left(I \pi R^{2} \gamma_{\mathrm{lg}}\right)\right)$

$$
\begin{aligned}
\tilde{G}= & \frac{1-f}{\gamma_{\mathrm{lg}} \pi R^{2}}\left[\left(p_{1}-p_{l}\right) V_{\text {in }}+\left(p_{0}-p_{1}\right) V_{0}+p_{0} V_{0} \ln \frac{p_{1}}{p_{0}}\right] \\
& +(1-f)\left(2 \frac{1-\cos \theta}{\sin ^{2} \theta}-\frac{2 h}{R} \cos \theta_{e}-1\right)
\end{aligned}
$$

Equation 7 describes the change of the free energy due to the protrusion of the liquid into the pores under hydrostatic pressure, with respect to the reference state (c.f., Figure $2 b$ ). $\tilde{G}$ is a function of the parameters $\theta, h$, and $p_{1}$ at a given liquid pressure $p_{1}$, and determines the pinning and depinning of TCL at the pore tops. A minimization of $\tilde{G}$ will lead to the equilibrium states of the system under the different liquid pressures. 


\section{MORPHOLOGIES OF LIQUID-AIR INTERFACE}

In this section, we consider the equilibrium morphologies of the liquid-air interface. Setting $\partial \tilde{G} / \partial p_{1}=0$ and $\partial \tilde{G} / \partial \theta=0$ yields

$$
V_{0} p_{0}=V_{1} p_{1}
$$

and

$$
p_{1}-p_{1}=\frac{2 \sin \theta}{R} \gamma_{\mathrm{lg}}
$$

Equation 8 is the ideal gas law at a constant temperature. Equation 9 is the Young-Laplace equation relating the capillary pressure difference to the meniscus curvature radius. If $\theta$ is in the range of $\left[\theta_{\mathrm{rec}}-\pi, \theta_{\mathrm{adv}}-\pi / 2\right]$ (where $\theta_{\mathrm{rec}}$ and $\theta_{\mathrm{adv}}$ are the receding and advancing contact angles, respectively), the TCL will remain pinned at the corners. ${ }^{11}$ That is, $h=0$, as illustrated in Figure 3a,b. Combining eq 3 at $h=0$ and eqs $8-9$ yields

$$
p_{l}-\frac{1}{1-\frac{R / H}{3 \sin ^{3} \theta}\left(2-3 \cos \theta+\cos ^{3} \theta\right)} p_{0}=\frac{2 \gamma_{\mathrm{lg}} \sin \theta}{R}
$$

Equation 10 gives an illustration of the equilibrium states as shown in Figure 3a,b with TCL still pinned at the top. When the liquid pressure is relatively lower than the initial air pressure, the liquid-air interface will protrude upward into the liquid and form a bubble, or even spread out on the upside of the substrate when the receding contact angle is reached, ${ }^{11}$ as shown in Figure $3 \mathrm{~b}$. Otherwise, when the liquid pressure is higher than the initial air pressure, the liquid-air interface will protrude downward into the pore, or even depin from the corner and slip into the pore with a constant advancing CA until a new equilibrium state is reached, as shown in Figure 3c.

In this work, we mainly consider the case that the initial air pressure $p_{0}$ is lower than the hydrostatic pressure. Setting $\partial \tilde{G}$ / $\partial h=0$, we have

$$
p_{1}-p_{1}=-\frac{2 \cos \theta_{\mathrm{e}}}{R} \gamma_{\mathrm{lg}}
$$

If the pore is open at the other end, the air pressure will remain constant, that is, $p_{1}=p_{0}$, and then eq 11 is usually referred to as the depinning transition criteria for the wetting on open structure surfaces. $^{13,14,25}$ For the pillared substrates, $R$ should be replaced by the effective capillary radius $R_{\mathrm{eff}}^{\mathrm{c}}$ defined by ${ }^{14}$

$$
R_{\text {eff }}^{c}=\frac{2 A}{C}
$$

where $A$ is the horizontal projected interface area and $C$ is the arc length of the three-phase contact line. Comparison of eqs 9 and 11 yields $\theta=\theta_{\mathrm{e}}-\pi / 2$. Then, by combining eqs 3,8 , and 11 , we obtain

$$
\begin{aligned}
p_{1} & -\frac{p_{0}}{1-h / H+\frac{R / H}{3 \cos ^{3} \theta_{\mathrm{e}}}\left(2-3 \sin \theta_{\mathrm{e}}+\sin ^{3} \theta_{\mathrm{e}}\right)} \\
& =-\frac{2 \gamma_{\mathrm{lg}} \cos \theta_{\mathrm{e}}}{R}
\end{aligned}
$$

The equilibrium state illustrated by eq 13 is shown in Figure 3c. Equations 10 and 13 provide a full description of the equilibrium morphologies of the liquid-air interface depending on the liquid and initial air pressures. Substituting eqs 8 and 10 or eqs 8 and 13 into eq 7 yields the minimum of $\tilde{G}$ as a function of the liquid pressure $p_{1}$. We denote this minimum of $\tilde{G}$ as $\tilde{G}_{\min }$. In the next section, we will discuss the wetting stabilities of the equilibrium states.

\section{STABILITIES OF EQUILIBRIUM STATES}

The equilibrium state described by eq 10 is the $\mathrm{CB}$ state the actual shape of the liquid-air interface before depinning considered. Equation 13 describes a new equilibrium state, which happens after the TCL depinning from the tops. The new equilibrium is reached as a result of the increasing air pressure by the liquid pressurization. It can easily be estimated from eq 7 that both states of eqs 10 and 13 are local stable ones. The global stabilities of these equilibrium states can simply be analyzed by an energy comparison with the Wenzel state. The collapse of the liquid-air interface will lead to the Wenzel state (c.f., Figure $3 \mathrm{~d}$ ), and $\tilde{G}_{\text {min }}$ in the Wenzel state is expressed as

$$
\begin{aligned}
\tilde{G}_{\min }= & -(1-f)\left(\frac{2 H}{R}+1\right) \cos \theta_{e}+f-1 \\
& +\frac{1-f}{\gamma_{\lg }}\left(p_{0}-p_{l}\right) H
\end{aligned}
$$

For a given solid material and surface structure, eq 14 is influenced by the hydrostatic pressure $p_{1}$.

Note that the real surface is hysteretic and that the motion of TCL during the wetting transition from the CB to Wenzel state does not change signs. $\theta_{\mathrm{e}}$ in $\tilde{G}_{\text {min }}$ should be replaced by $\theta_{\text {adv }}{ }^{26}$ Consider a surface structure, e.g., $R=3.0 \mu \mathrm{m}, H=9.5 \mu \mathrm{m}$, and $\theta_{\text {adv }}=120^{\circ}$. It is noted that the solid fraction $f$ varies with the lattice constant $L ; f$ only influences the scale of $\tilde{G}$, and the equilibrium pressures are independent of $f$ (c.f., eqs 7,10 , and 13). Therefore, for the sake of brevity, $f=0.5$ is chosen. The liquid is water with surface tension $\gamma_{\mathrm{lg}}=0.0728 \mathrm{~N} / \mathrm{m}$, and the atmosphere pressure $p_{0}$ is set to $101.3 \mathrm{kPa}$. Then, according to eq 7, $\tilde{G}_{\text {min }}$ (in different equilibrium states illustrated by eqs 10 , 13 , and 14 , respectively) as a function of $p_{1} / p_{0}$ is shown in Figure 4. From Figure 4, we can see that there are two critical pressures $\mathrm{P} 1$ and $\mathrm{P} 2$. P1 represents the pressure at which the free energies of the $\mathrm{CB}$ and Wenzel states are equal. P2 is the pressure at which the sagging angle of the meniscus $\theta$ reaches the value of $\theta_{\text {adv }}$, as shown by the dashed line in Figure 3a. After

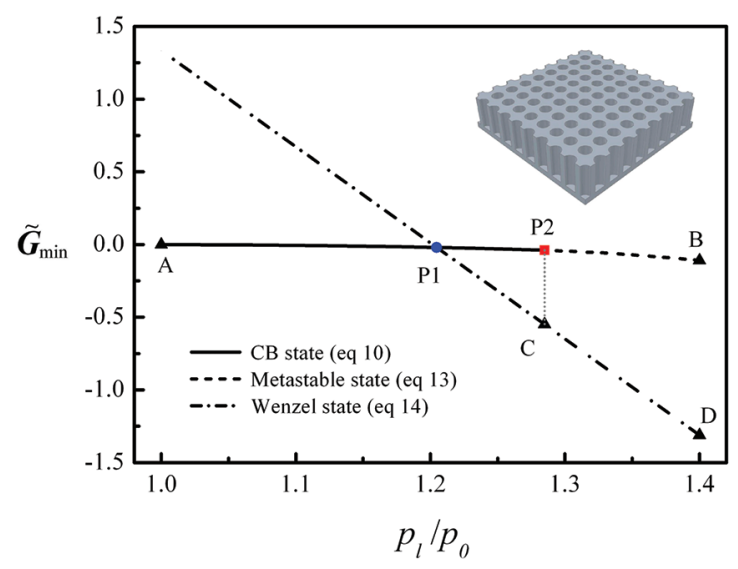

Figure 4. Plots of $\tilde{G}_{\min }$ as a function of $p_{1} / p_{0}$ for different equilibrium states on surfaces with patterned pores (c.f., the insert). P1, the pressure at which the Wenzel state becomes lower energy than the $\mathrm{CB}$ state; P2, the pressure at which the depinning happens. 
P2, the TCL starts to depin from the top. P1 and P2 divide the wetting behaviors of submersed surfaces under pressure into three different kinds. First, at low hydrostatic pressure, the $\mathrm{CB}$ state (from A to P1, illustrated by eq 10 and Figure 3a) with lower free energy than that of the Wenzel state, is stable and always preferential in general cases. Second, as the hydrostatic pressure increases, the Wenzel state has a lower energy than that of the $\mathrm{CB}$ state after $\mathrm{P} 1$. Third, when the pressure increases further beyond P2, the TCL depinning will happen (c.f., the dashed line in Figure 3a). Though the free energy of the Wenzel state from $\mathrm{P} 1$ to $\mathrm{C}$ is relatively lower, the CB state from $\mathrm{P} 1$ to $\mathrm{P} 2$ is still more preferential, due to the stabilization given by the TCL pinning, and a large disturbance is needed to induce the transition from the $\mathrm{CB}$ to Wenzel states. This is the reason it is generally accepted that the wetting transition appears on an open-structured surface when the depinning happens. ${ }^{11,14,25}$

$\mathrm{By}$ comparison with the $\mathrm{CB}$ state (from $\mathrm{P} 1$ to $\mathrm{P} 2$ ), the depinned metastable state (from P2 to B as illustrated by eq 13 and Figure 3c) can withstand relatively smaller disturbances. This is because this state has higher free energy than the Wenzel sate (from $\mathrm{C}$ to $\mathrm{D}$ ). On the other hand, the liquid pressure, which the structured surface can support, will be significantly increased by achieving the metastable state from $\mathrm{P} 2$ to $\mathrm{B}$. According to eq 13, $p_{1}$ increases from $1.28 \cdot p_{0}$ to 1.34. $p_{0}$, when $h$ changes from 0 to $0.05 H$ for a cylindrical pore with $R=3 \mu \mathrm{m}$ and $\theta_{\mathrm{adv}}=120^{\circ}$ (c.f., Figure 4). This means that the pressure difference (i.e., $p_{1}-p_{0}$ ) is increased by $20 \%$. This increase will be more pronounced for the superhydrophobic surfaces with a large area of menisci. ${ }^{13}$ However, until now, no theories or direct experiments have paid much attention to this depinned metastable state. In section 5, by comparing our theory with available experiments in the literature, for the first time we will show that an equilibrium state after depinning (i.e., the depined metastable state) on submersed superhydrophobic surfaces is possible. In section 6, we will discuss how to improve the stability of the metastable state.

From the above theoretical analysis, it is seen that $\tilde{G}_{\min }$ is influenced by the solid fraction $f$ and the hydrostatic pressure $p_{1} / p_{0}$ (c.f., Figure 4). In order to analyze the influence of $f$ and $p_{1} / p_{0}$ on $\tilde{G}_{\min }$, the contour plots of $\tilde{G}_{\min }$ as functions of $f$ and $p_{1} /$ $p_{0}$ on a surface with patterned cylindrical pores of constant radius and depth (e.g., $R_{\mathrm{p}}=3.0 \mu \mathrm{m}$ and $H=9.5 \mu \mathrm{m}$ ) are shown in Figure 5. Due to the assumption of constant pore size, the critical pressures $\mathrm{P} 1$ and $\mathrm{P} 2$ are independent of $f$. The solid



Figure 5. Contours of $\tilde{G}_{\min }$ as functions of $f$ and $p_{1} / p_{0}$ on surfaces with patterned pores (c.f., Figure 2a). fraction $f$ of the structured surface with pore patterns on a square lattice (c.f., Figure 4$)$ is in the range of $f \in(0.21,1.0)$. From Figure 5, we can see that $\tilde{G}_{\text {min }}$ increases with decrease of $p_{1} / p_{0}$ and increase of $f$. The whole phase diagram is divided into three regions: Region $\mathrm{I}$ is the pressure range from $\mathrm{A}$ to $\mathrm{P} 1$, in which the CB state is generally stable; Region II is the pressure range from $\mathrm{P} 1$ to $\mathrm{P} 2$ (or $\mathrm{C}$ ), in which the $\mathrm{CB}$ state (from $\mathrm{P} 1$ to $\mathrm{P} 2$ ) has higher free energy than the Wenzel sate from P1 to C; and Region III is from P2 to B (or from C to D), in which the depinned metastable state (from P2 to B) has much higher free energy than the Wenzel state from (C to D, c.f., Figure 4). Given a specific submersed surface structure and a given hydrostatic pressure, we can determine the wetting stability from this phase diagram.

Noted that the pillared surfaces are generally used for the drag reduction applications, because a large and continued liquid-air interface can be obtained on such surfaces for minimizing drag. ${ }^{18}$ In the following, we will extend the formulation developed above to analyze the wetting behaviors of submersed pillared surfaces, and compare our theoretical results with the existing experimental ones in the literature.

\section{WETTING STABILITY OF PILLARED SURFACES}

Recently, wetting states and transitions on pillared surfaces have received much attention. For example, Forsberg et al. ${ }^{13}$ carried out experiments to study the wetting transition on submersed superhydrophobic surfaces and demonstrated that a closed film of trapped air helps stabilize the Cassie-Baxter state, especially in the low pillar densities. They used pillarstructured surfaces made of $\mathrm{PE}$ (polyethylene) with $\theta_{\mathrm{adv}}=$ $103.5^{\circ}$, and fluoropolymer-coated PE with $\theta_{\mathrm{adv}}=122.1^{\circ}$. In their experiment, those surfaces were fully immersed in water, whose pressure was raised or lowered until the wetting transitions happened, and they recorded the collapse pressures on the pillared surfaces with different solid fractions.

To better illustrate the wetting stability on the submersed substrates, it is necessary to carry out a comparison between the present theory and the experiment results (e.g., those obtained by Forsberg et al. ${ }^{13}$ ). Before that, we need to extend the formulation developed in the above sections so as to analyze the behavior on submersed pillared surfaces. Noted that Forsberg et al. ${ }^{13}$ investigated the influence of the pillar shape and arrangement as well, e.g., circular and square pillars in a square or hexagonal array. Their results show that the variation of pillar shapes and arrangements do not show much difference (any difference is within the uncertainty of the measurement ${ }^{13}$ ) with similar pillar size and solid fraction. Therefore, without loss of generality, we investigate a surface structure of circular pillars on a periodic square lattice (shown in Figure 6a). Other structures can be investigated following the similar procedure.

5.1. Stabilities of Equilibrium States. The pillared surface can be approximately equivalent to a pore-patterned one with corresponding effective geometric and capillary radii. The effective geometric radius means that the liquid volume protruding into each effective pore is approximately equal to that protruding between the four nearby pillars. For pillars on a square lattice (as shown in Figure 6b), the effective geometric radius can be approximately expressed as

$$
R_{\mathrm{eff}}^{\mathrm{g}}=\left(\sqrt{\frac{\pi}{2 f}}-1\right) R_{\mathrm{p}}
$$




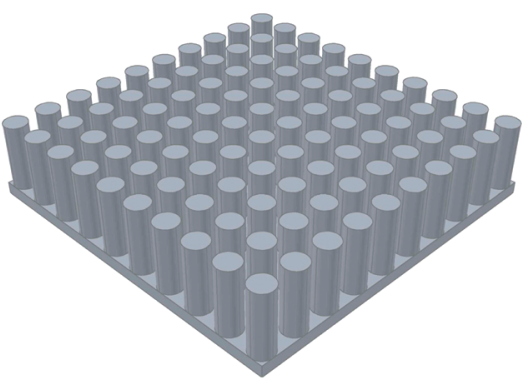

(a)

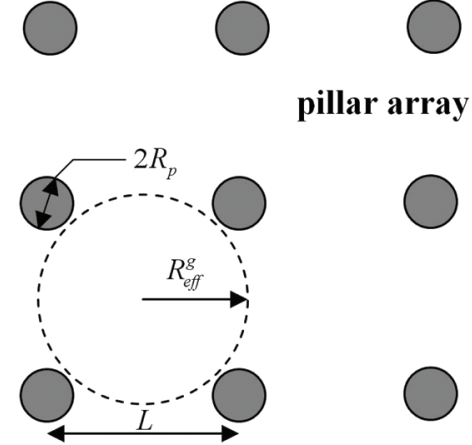

(b)

Figure 6. (a) Structured surface patterned with pillars. (b) Schematic illustrating the effective geometric radius $R_{\text {eff }}^{\mathrm{g}}$ for a structured surface with a pillar radius $R_{\mathrm{p}}$ on a periodic square lattice of constant $L$ (top view).

where $R_{\mathrm{p}}$ is the pillar radius. A specific form of eq 12 gives the effective capillary radius for pillars on a square lattice

$$
R_{\text {eff }}{ }^{\mathrm{c}}=\frac{1-f}{f} R_{\mathrm{p}}
$$

Note that the values of $R_{\mathrm{eff}}^{\mathrm{g}}$ and $R_{\mathrm{eff}}^{\mathrm{c}}$ may differ greatly from each other, depending on the surface structure and solid fraction. With eqs $15-16$, the normalized total free energy $\tilde{G}$ for pillared surfaces as functions of $\theta, h, p_{1}$, and $p_{1}$ can be easily obtained, following the same procedure to get eq 7 . The derivation of $\tilde{G}$ with respect to different parameters leads to the equilibrium states. The analysis shows that the states of eqs 10 and 13 also exist on pillared surfaces. $\tilde{G}_{\min }$ on pillared surfaces is given by $\tilde{G}$ in the equilibrium states (e.g., the $\mathrm{CB}$, metastable and Wenzel states). Especially, eq 13 for the pillared substrates can be expressed as

$$
\begin{aligned}
p_{1}- & p_{0} /\left[1-h / H+\frac{f}{(1-f) R_{\mathrm{p}}{ }^{2} H} \frac{\left(R_{\mathrm{eff}}^{\mathrm{g}}\right)^{3}}{3 \cos ^{3} \theta_{e}}\right. \\
& \left.\left(2-3 \sin \theta_{e}+\sin ^{3} \theta_{e}\right)\right] \\
= & -\frac{2 \gamma_{\mathrm{lg}} \cos \theta_{e}}{R_{\mathrm{eff}}^{\mathrm{c}}}
\end{aligned}
$$

Note that the height of those pillars is $H$. Equation 17 gives the collapse pressure on submersed pillared surfaces, and its accuracy depends on the approximation made by eqs $15-16$.

In accordance with the experiments of Forsberg et al., ${ }^{13}$ we consider a pillared surface with $R_{\mathrm{p}}=3.0 \mu \mathrm{m}$ and submersed in water. To understand the energy states on the pillared surface clearly, we present a free energy comparison of different equilibrium states (e.g., the $\mathrm{CB}$, metastatble and Wenzel states) in Figure 7, with respect to two different $\theta_{\text {adv }}$ (i.e., $103.5^{\circ}$ and $\left.122.1^{\circ}\right)$. The solid-fraction range is from 0.1 to 0.5 in the experiment of Forsberg et al. ${ }^{13}$ Here, an intermediate value of $f=0.3$ is chosen as an example to illustrate the wetting stabilities on pillared surfaces. The critical pressures P1 and P2 (c.f., Figure 4) are also included. For $\theta_{\mathrm{adv}}=103.5^{\circ}$, the plots in Figure 7 are similar to Figure 4, which are divided into three parts. In the first part, the $\mathrm{CB}$ state (from A to $\mathrm{P} 1$ ) is generally stable at low pressure; in the second part, the $\mathrm{CB}$ state (between P1 and P2) has higher free energy than the Wenzel state (from P1 to C); and in the last part, the metastable state

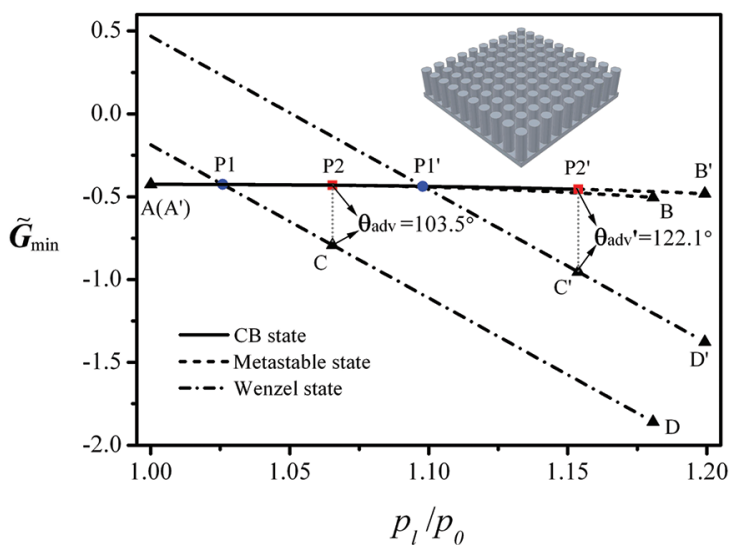

Figure 7. Plots of $\tilde{G}_{\min }$ as a function of $p_{1} / p_{0}$ for different equilibrium states on pillared surfaces (c.f., the inset, $R_{\mathrm{p}}=3.0 \mu \mathrm{m}$ and $H=9.5 \mu \mathrm{m}$ ) with respect to $\theta_{\mathrm{adv}}=103.5^{\circ}$ and $\theta_{\mathrm{adv}}{ }^{\prime}=122.1^{\circ}$.

(from P2 to B) exists but has much higher free energy than the Wenzel state (from $C$ to $D$ ).

It is noted that Forsberg et al. ${ }^{13}$ used the fluoropolymer coating with higher hydrophobicity $\left(\theta_{\text {adv }}=122.1^{\circ}\right)$ in their experiment. This is because it is known that materials with high hydrophobicity can improve the stability of structured surfaces. In order to compare with the results with $\theta_{\mathrm{adv}}=103.5^{\circ}$, we plot the free energies in different equilibrium states with $\theta_{\mathrm{adv}}=$ $122.1^{\circ}$ in Figure 7 as well. The points with primes (e.g., A', P1', $\left.\mathrm{P} 2{ }^{\prime}, \mathrm{B}^{\prime}, \mathrm{C}^{\prime}, \mathrm{D}^{\prime}\right)$ denote those with $\theta_{\mathrm{adv}}=122.1^{\circ}$ (c.f., Figure 7). It is shown that the high hydrophobicity can enhance the critical pressures (e.g., from P1 to P1'; from P2 to P2'). This enhancement means that structured surfaces with the higher hydrophobicity can withstand larger liquid pressure.

Similar to Figure 5, the contour plots of $\tilde{G}_{\text {min }}$ as functions of $f$ and $p_{1} / p_{0}$ on the pillared surfaces (c.f., Figure 6a) are shown in Figure 8 . The radius and height of those pillars are kept constant, e.g., $R_{\mathrm{p}}=3.0 \mu \mathrm{m}$ and $H=9.5 \mu \mathrm{m}$. The range of $f$ is chosen as $f \in[0.1,0.5]$, in which the validation of our theory has been confirmed by comparison with numerical and experimental results in the following section. The dependence of the effective geometric and capillary radii on $f$ influences the critical pressures $\mathrm{P} 1$ and P2. In fact, Figure 8a,b shows phase diagrams of $\tilde{G}_{\min }$ with $f$ and $p_{1} / p_{0}$. Given a specific submersed surface structure $(f)$ and a given hydrostatic pressure $\left(p_{1} / p_{0}\right)$, we can determine the wetting state from those diagrams. Likewise, the whole phase diagrams in Figure 8 are also divided 
(a)

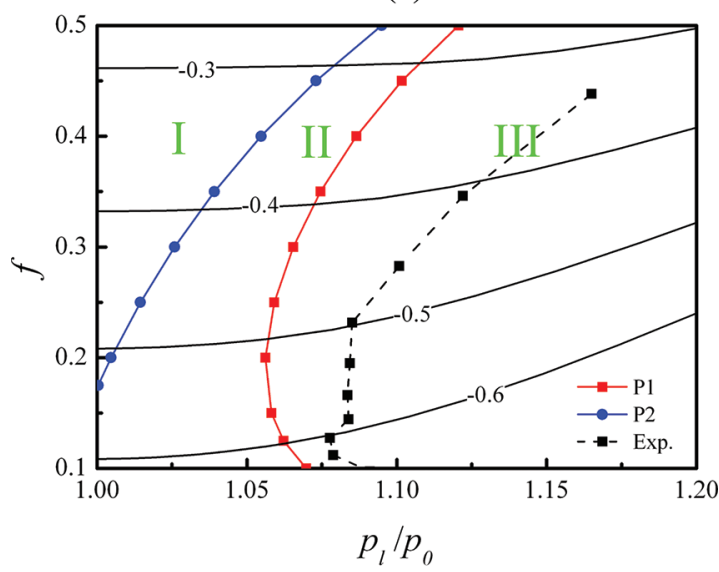

(b)

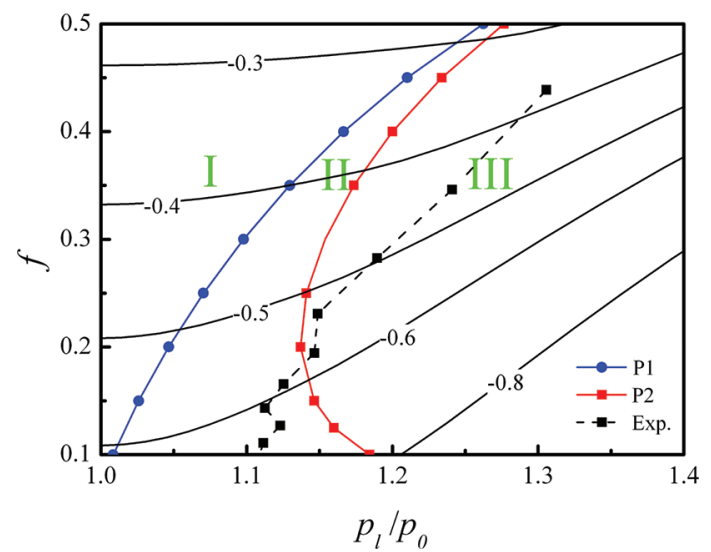

Figure 8. Contours of $\tilde{G}_{\min }$ as functions of $f$ and $p_{1} / p_{0}$ on pillared surfaces (c.f., Figure 6). The experimental results are from Forsberg et al.: ${ }^{13}$ (a) $\theta_{\text {adv }}=103.5^{\circ}$, (b) $\theta_{\text {adv }}=122.1^{\circ}$.

into three regions: Region I, Region II, and Region III (c.f., Figure 5 and Section 4 for detailed descriptions). From Figures 7 and 8 , we can easily know that on pillared surfaces, in region $\mathrm{I}$, the $\mathrm{CB}$ state is very robust and the transition to the Wenzel state can barely happen; in region II, the transition from the $\mathrm{CB}$ to Wenzel states can only happen under relatively large disturbances, because the TCL pinning assists its stability; and in region III, relatively low disturbances can induce the transition from the depinned metastable to Wenzel states. Given a specific submersed surface structure $(f)$ and a given hydrostatic pressure $\left(p_{1} / p_{0}\right)$, we can determine the wetting state from those diagrams. For example, a structure of $f=0.3$ under $p_{1}=1.1 p_{0}$ is in a metastable state after depining (i.e., in region III in Figure 8a) when $\theta_{\text {adv }}=103.5^{\circ}$; whereas a structure of $f=$ 0.3 under $p_{1}=1.1 p_{0}$ is in a $\mathrm{CB}$ state before depinning (i.e., in region II in Figure 8b) when $\theta_{\mathrm{adv}}=122.1^{\circ}$. This further proves that an increase of the hydrophobicity (e.g., $\theta_{\text {adv }}$ from $103.5^{\circ}$ to $122.1^{\circ}$ ) not only helps the surface to support high pressure, but also improves the wetting stability. To confirm the above analysis, a comparison with the experimental results in the literature is constructed.

5.2. Comparison with Experimental Results. As mentioned above, several experiments have been carried out on wetting transitions on submersed structured surfaces. The experimental results from Forsberg et al. ${ }^{13}$ are shown in Figure $8 \mathrm{a}-\mathrm{b}$. It is clearly seen that almost all results fall in Region III, which means that the TCL has slipped into the cavity at a certain distance (c.f., Figure 3c). There are several exceptional experiment results on the pillared surfaces with $\theta_{\mathrm{adv}}=122.1^{\circ}$, in which the collapse appeared before the depinning, falling in Region II. This is most probably because certain disturbances appeared during their experiments, which exceeded the energy barrier between the Wenzel and CB states. Note that in Region II the Wenzel state has a lower free energy than the CB sate. Thus, though the CB state is preferential due to the TCL pinning, the transition may happen under certain disturbances.

From the above free-energy analysis, it is shown that the experimental results of Forsberg et al. ${ }^{13}$ were obtained from the metastable states (c.f., Region III). In order to confirm that further, the collapse pressure (obtained by eq 17) as a function of the solid fraction $f$ (together with the experimental results from Forsberg et al. ${ }^{13}$ ) is plotted in Figure 9. When $h / H=0, p_{1}$

(a)

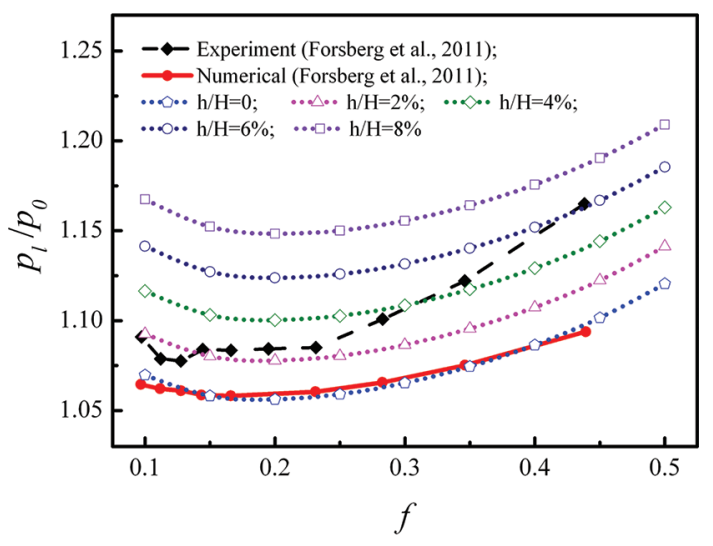

(b)

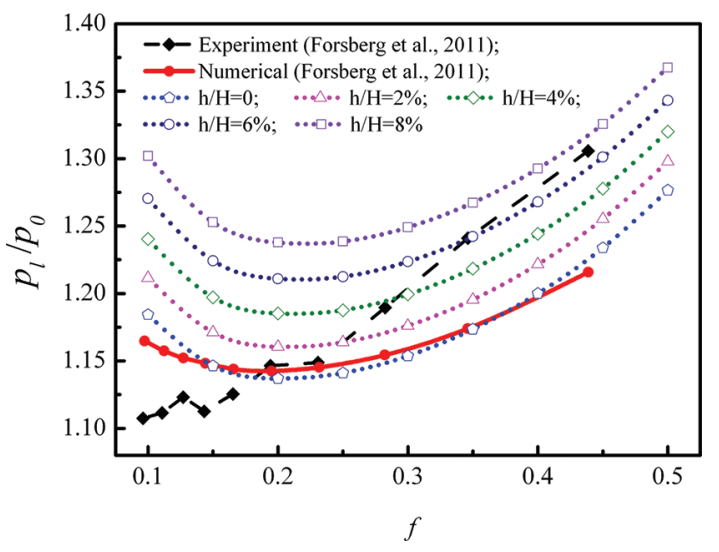

Figure 9. Contour plots of the collapse pressure $p_{1}$ (calculated by eq 17) as a function of the solid fraction $f$ on the pillared substrates $\left(R_{\mathrm{p}}=\right.$ $3.0 \mu \mathrm{m}$ and $H=9.5 \mu \mathrm{m}$ ): (a) $\theta_{\mathrm{adv}}=103.5^{\circ}$, (b) $\theta_{\mathrm{adv}}=122.1^{\circ}$.

obtained by eq 17 is the depinning transition pressure, which is in a good agreement with the numerical results of Forsberg et al. ${ }^{13}$ within the tested range of solid fraction (i.e., $0.1-0.45$ ), as shown in Figure 9a,b. At a low solid fraction, the depinning pressure is close to the experimental one. As the solid fraction increases from 0.1 to 0.45 , the predicted depinning pressure does not change much, but the experimental one changes a lot, even $1 \times$ higher. A comparison between plots of eq 17 (Figure $9 \mathrm{a}, \mathrm{b}$ and the experimental results) implies that the depinned liquid-air interface has slipped into the cavity at a certain 
distance $h$ (as illustrated in Figure 3c). For example, for the case of $\theta_{\text {adv }}=103.5^{\circ}$ at $f=0.3$, a relative protrusion depth $(h / H$ $\sim 4 \%)$ is obtained, and thus, an air pressure increase $\sim 4 \mathrm{kPa}$ results. This contributes about $40 \%$ to the pressure difference (i.e., $p_{1}-p_{0}$ ) just before the collapse of the liquid-air interface.

There is another experiment to investigate wetting transitions on submersed superhydrophobic surfaces by using the technique of transmission diffraction patterns. ${ }^{10}$ Their starting point is that the change of the diffraction spot intensity shows how deep the meniscus has protruded into the cavity, and the disappearance of the diffraction means that a transition from a $\mathrm{CB}$ to Wenzel state has happened. The structured surface used is a PDMS grating of $10-\mu \mathrm{m}$-diameter and $50-\mu \mathrm{m}$ height pillars on a pseudotriangle lattice, and the solid fraction $f$ is about 0.19. Given the contact angle on a flat PDMS of $\sim 105^{\circ}$, the depinning pressure (i.e., P2) on their grating is about $1.028 p_{0}$, according to eqs $16-17$. By gradually increasing the liquid pressure from $p_{0}$ to a maximum value (e.g., $1.05 p_{0}$ or $\left.1.06 p_{0}\right)$, and then decreasing back to $p_{0}$, Lei et al. ${ }^{10}$ observed reversible diffraction pattern when the maximum pressure is lower than $1.06 p_{0}$. The diffraction spot intensity In (zeroorder) as a function of liquid pressure obtained by Lei et al. ${ }^{10}$ is normalized by its starting value $\operatorname{In}_{0}$ (when $p_{1}=p_{0}$ ), and we replot it in Figure 10. According to eq 17, the corresponsive

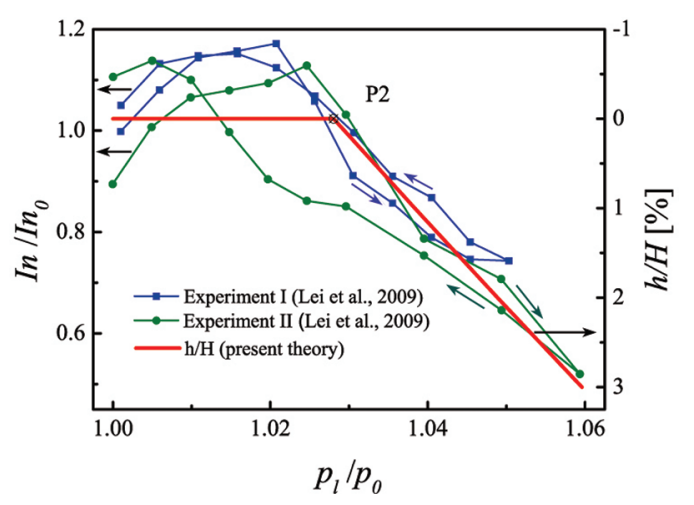

Figure 10. Correlation between the relative protrusion depth $h / H$ (present theory) and normalized diffraction spot intensity $\mathrm{In} / \mathrm{In}_{0}$ (experimental results from ref 10) as functions of the liquid pressure $p_{1} / p_{0}$. Experiment I: the liquid pressure increases from $p_{0}$ to $1.05 p_{0}$ and then deceases to $p_{0}$. Experiment II: the liquid pressure increases from $p_{0}$ to $1.06 p_{0}$ and then deceases to $p_{0}$.

relative protrusion depths $h / H$ are calculated and plotted in Figure 10 to illustrate the correlation between the diffraction spot intensity and the protrusion depth. It can be clearly seen that, when $p_{1}$ is smaller than the depinning pressure, the TCL is still pinned at the corner, i.e., $h / H=0$, and the diffraction spot intensity varies slightly as the increase of $p_{1}$. A rapid decay of the diffraction intensity appears when $p_{1}$ is higher than P2. This is because the pressure higher than $\mathrm{P} 2$ induces the depinning of the TCL, and the protrusion depth into the cavities increases as the increase of $p_{1}$.

The good agreement between our theory and the experiments of Lei et al. ${ }^{10}$ reconfirms the existence of the metastable state after depinning. The depinning of TCL may not induce the failure of superhydrophobicity under water. It is possible that a metastable state exists after the TCL depinning, due to the increasing air pressure as the liquid protrusion into the cavities. Noted that our theory is based on pillars on a square lattice, which is different from the pseudotriangle lattice of Lei et al. $^{10}$ The good agreement between our theory and experiment of Lei et al. ${ }^{10}$ confirms the conclusion obtained by Forsberg et al. ${ }^{13}$ that the characteristic size of pillars and the solid fraction play more important roles than the pillar arrangement. The depinned metastable state raises the collapse hydrostatic pressure supported by a superhydrophobic surface. However, due to much higher free energy of the metastable state than the Wenzel state, the metastable state is not immune to external disturbances. Therefore, to utilize the advantage of the metastable state, it is necessary to strengthen its stability.

5.3. Comparison of Wetting Stability of Two Structured Surfaces. From Figures 4 and 7, we know that a large area of closed-air film after depinning from the tops is in a metastable state, and some disturbances may induce the local collapse of the film, as illustrated in the above sections. The local collapse of the closed-air film expands as the hydrostatic pressure gradually increases, as observed in the experiments of Forsberg et al. ${ }^{13}$ When the solid fraction is higher, the closedair film becomes more stable due to a larger arc length of the TCL per unit area, and thus, the liquid-air interface can sustain a higher disturbance and protrude deeper into the cavities. As shown in Figure 9a,b, the relative protrusion depth increases from $\sim 0 \%$ to $\sim 7 \%$, as the solid fraction increases from $\sim 0.1$ to $\sim 0.45$.

It is seen that closed-air films on substrates with patterns of individual cavities (c.f., Figure 2a) are in a much smaller area than those on the pillared substrates (c.f., Figure 6a). It can be expected that the air films trapped in patterns of cavities are much more stable than those in the pillared substrates with the same solid fraction in the low range, e.g., $f<0.5$. For example, for the two kinds of surfaces with the same lattice parameter, height and hydrophobicity, e.g., $L=10 \mu \mathrm{m}, H=9.5, \mu \mathrm{m}$ and $\theta_{\text {adv }}=120^{\circ}$ (c.f., Figures 2 and 6), at a solid fraction $f=0.3$, the depinning pressures (according to eqs 13 and 17) for the surfaces patterned with cavities and pillars are $1.225 \cdot P_{0}$ and $1.142 \cdot P_{0}$, respectively. When $h$ is $0.05 H$, the collapse pressures is $1.286 \cdot P_{0}$ (with cavities) and $1.120 \cdot P_{0}$ (with pillars), respectively. In both cases, the surface patterned with cavities can support relatively higher liquid pressure than that of the pillared one. That is, a more stable superhydrophobicity can be expected on substrates patterned with individual cavities.

However, in real applications, we also need a large fraction of continued liquid-air interface for minimizing drag in a high liquid pressure (and/or velocity) environment. In the following section, we will discuss how to obtain a metastable state on pillared surfaces with high stability.

\section{WETTING STABILITY OF HIERARCHICAL STRUCTURED SURFACES}

The comparison between our analytical results and experimental results in the literature in the above section confirms that a metastable state exists after the TCL depins from the tops (c.f., Figures 7-10). The metastable state helps the submersed superhydrophobic surface support higher liquid pressure. Increasing the stability of the metastable state will strengthen the superhydrophobicity. To maintain a metastable state, the TCL should be strongly pinned on the cavity sidewalls to avoid the collapse transition. Therefore, techniques assisting the pinning of TCL will help to obtain stable and large area liquid-air interfaces on structured surfaces. Inspired by the super antiwetting properties of lotus leaves and water strider legs, hierarchical structures are commonly used to improve the wetting stability of superhydrophobic surfaces. Various artificial 

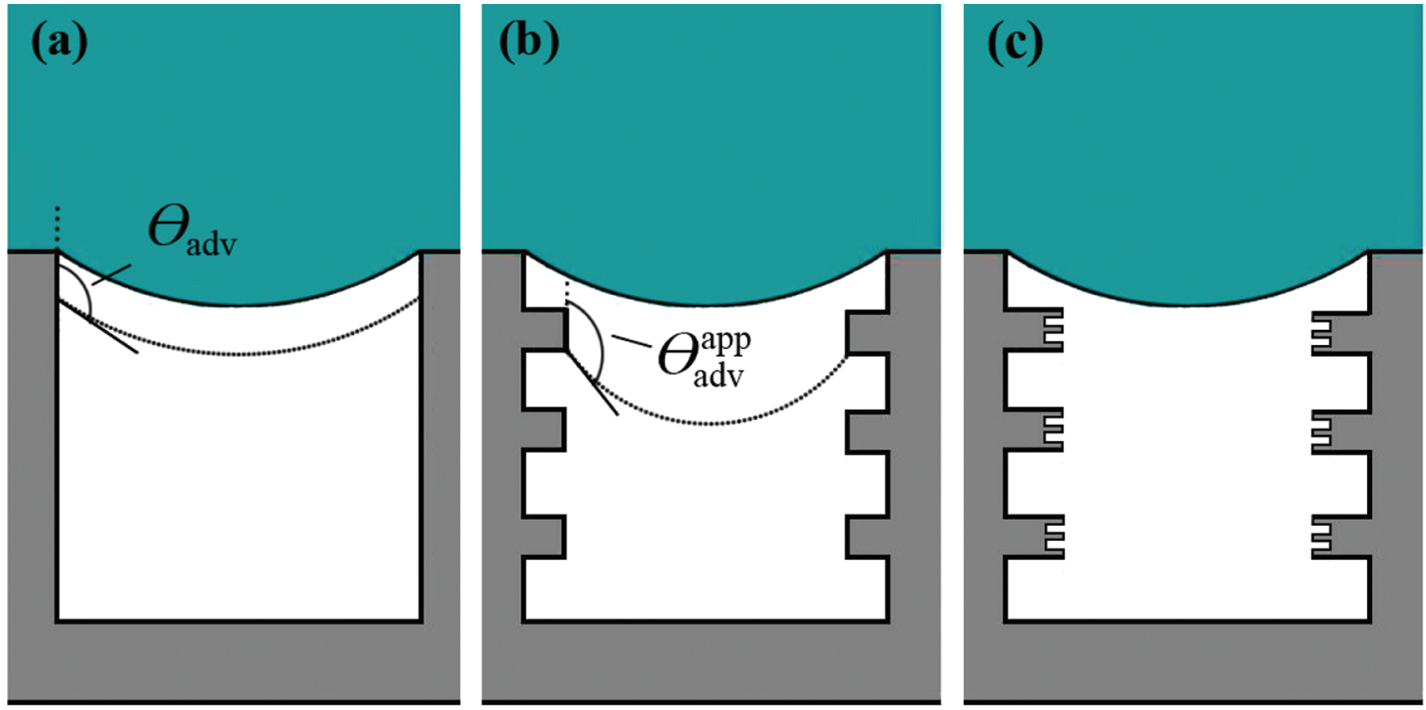

Figure 11. Schematic illustrating the pinning enhancement of the TCL by sublevel structures on the sidewalls. The dotted lines show the liquid-air interface in the metastable state (c.f., Figure 3c): (a) $\theta_{\text {adv }}$ on the smooth sidewalls, (b) larger $\theta_{\text {adv }}^{\text {app }}$ on the structured sidewalls, and (c) three-level structure obtained by modifying the tops of sublevel pillars.

hierarchical structures have been fabricated based on pillared surfaces by the methods of deposition and corrosion, and so forth. In the following, we will show how the hierarchical structures can be used to improve the stability on submersed pillared surfaces and the mechanisms.

It is known that microstructures or defects on substrates can significantly increase the wetting hysteresis due to TCL pinning. ${ }^{27}$ As illustrated in Figure 11, a hierarchical surface with substructured sidewalls helps for a stable liquid-air interface. This is because, when the TCL depins from the pillar tops, the sublevel structures will provide new pinning positions for the TCL. The metastable state after depinning can be strengthened by the sublevel structures. Thus, even if TCL has slipped at certain depth $h$ into the cavity, the liquid-air interface can still be in a quite stable state. From another point of view, the sublevel roughness on the sidewalls increases the wetting hysteresis, enabling the pinning of the TCL. Thus, a larger apparent advancing contact angle $\theta_{\text {adv }}^{\text {app }}$ than the original $\theta_{\text {adv }}$ on smooth sidewalls results, ${ }^{27}$ as shown in Figure $11 \mathrm{~b}$. A three-level or even higher level structure (as shown in Figure 11c) will further strengthen the pinning effect of the contact line. Namely, the TCL can slip into the cavity at a greater $h$ with a larger $\theta_{\mathrm{adv}}^{\mathrm{app}}$ due to the assistance given by the sublevel structures. In the following, a quantitative analysis will be given to determine the equilibrium states and their stabilities on submersed hierarchical surfaces.

The hierarchical pillared surfaces have the same configuration and structure size as shown in Figure 6 except that there are sublevel structures (e.g., pillars or grooves) on the sidewalls. Figure $11 \mathrm{~b}$ illustrates a two-level structure, which has a similar configuration as the surface structure of lotus leaves. On the surface of lotus leaves, there are first-level protrusions and sublevel pillars on these protrusions. ${ }^{17} \mathrm{~A}$ three-level structure can be obtained by further modification of the tops of those sublevel pillars, as shown in Figure 11c. For simplification, we consider a two-level structure as shown in Figure 11b. The analysis of three- or higher-level structures (see Figure 11c) is similar. Following the procedure as described in sections 2 and 5 , the total free energy for a submersed hierarchical pillared surface can be easily obtained. Minimizing the total free energy will lead to the equilibrium states. The metastable state after depinning can be expressed as

$$
\begin{aligned}
p_{l}- & p_{0} /\left[1-h / H+\frac{f}{(1-f) R_{p}^{2} H} \frac{\left(R_{\mathrm{eff}}^{\mathrm{g}}\right)^{3}}{3 \cos ^{3} \theta_{e}^{\mathrm{app}}}\right. \\
& \left.\left(2-3 \sin \theta_{\mathrm{e}}^{\mathrm{app}}+\sin ^{3} \theta_{\mathrm{e}}^{\mathrm{app}}\right)\right] \\
= & -\frac{2 \gamma_{\mathrm{lg}} \cos \theta_{\mathrm{e}}^{\mathrm{app}}}{R_{\mathrm{eff}}^{\mathrm{c}}}
\end{aligned}
$$

where $\theta_{\mathrm{e}}^{\mathrm{app}}$ is the apparent equilibrium contact angle on the structured sidewalls. For a quasistatic process of wetting transition from a $C B$ to Wenzel state, $\theta_{\mathrm{e}}^{\text {app }}$ in eq 18 should be replaced by the apparent advancing contact angle $\theta_{\text {adv }}^{\text {app }}{ }^{26}$ and $\theta_{\mathrm{adv}}^{\text {app }}$ is expressed by a modified CB equation ${ }^{1,28,29}$

$$
\theta_{\mathrm{adv}}^{\mathrm{app}}=f_{\mathrm{sw}} \cos \theta_{\mathrm{adv}}+f_{\mathrm{sw}}-1
$$

Here, $f_{\text {sw }}$ is the solid fraction of the structured sidewalls, and $\theta_{\mathrm{adv}}^{\mathrm{app}}$ represents the ground state of an advancing contact line along the sidewalls. ${ }^{29}$ The analysis of wetting stability on hierarchical structured surfaces can be achieved by following the procedure shown in section 5 .

With comparison of eqs 17 and 18, we know that the influence of sublevel-structured sidewalls is to give a higher $\theta_{\mathrm{adv}}^{\mathrm{app}}$ than $\theta_{\text {adv }}$. For example, given a structured sidewall with $f_{\mathrm{sw}}=0.3$ and $\theta_{\text {adv }}=103.5^{\circ}, \theta_{\mathrm{adv}}^{\text {app }}$ can be as high as $\sim 140^{\circ}$, according to eq 19. As described in sections 4 and 5 , the critical pressure P1 can be calculated by equalizing the free energies of $\mathrm{CB}$ and Wenzel states, and P2 can be obtained by letting $h=0$ in the formula of the collapse pressure (eq 17 or 18) (i.e., the depinning presure). In fact, P1 and P2 reflect the pressure-withstanding ability of superhydrophobic surfaces, and they are plotted in Figure 12 as a function of $\theta_{\text {adv }}$. From Figure 12, we can see that both $\mathrm{P} 1$ and $\mathrm{P} 2$ are significantly elevated as the increase of $\theta_{\mathrm{adv}}$. For example, an increase of $\theta_{\text {adv }}$ from $103.5^{\circ}$ to $140^{\circ}$ induces the elevation of P1 from 1.03 to 1.16 and $\mathrm{P} 2$ from 1.07 to 1.24 (as shown by the dashed lines $\mathrm{A}$ and $\mathrm{B}$ in Figure 12). The 


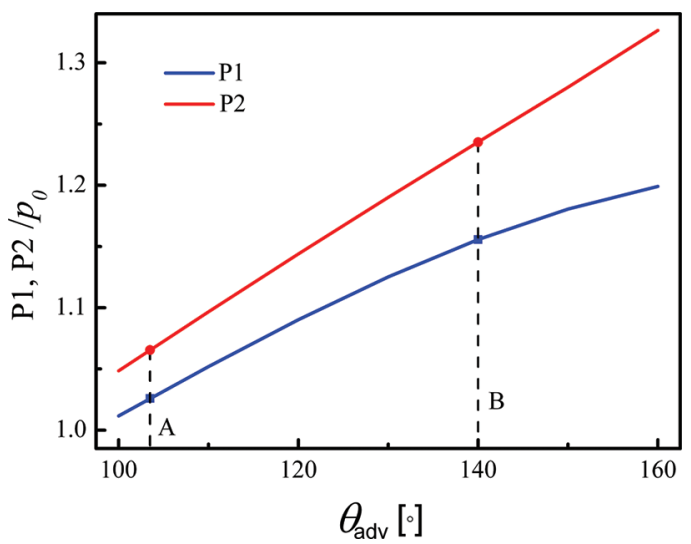

Figure 12. Plots of the critical pressures $\mathrm{P} 1$ and $\mathrm{P} 2$ as a function of $\theta_{\mathrm{adv}}$ $\left(R_{\mathrm{p}}=3.0 \mu \mathrm{m}, H=9.5 \mu \mathrm{m}\right.$, and $\left.f=0.3\right)$. Dashed lines A and B show the positions of $\theta_{\mathrm{adv}}=103.5^{\circ}$ and $\theta_{\mathrm{adv}}=140^{\circ}$, respectively.

elevation of P1 and P2 means that the stability of those surfaces has been improved.

We also plot the collapse pressure (i.e., $p_{1} / p_{0}$ by eq 18 ) as a function of $h / H$ on a pillared surface of solid fraction $f=0.3$ (with different $\theta_{\mathrm{adv}}^{\mathrm{app}}$ ), as shown in Figure 13. It is clearly shown

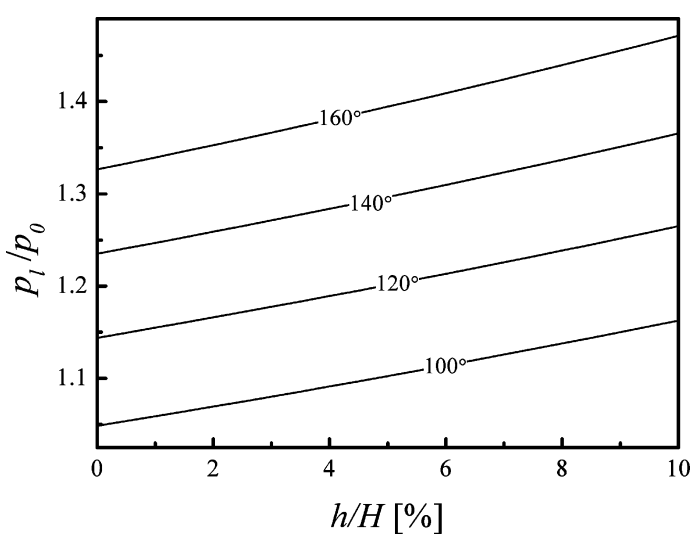

Figure 13. Plots of $p_{1} / p_{0}$ (calculated by eq 17) as a function of $h / H$ with different $\theta_{\mathrm{adv}}^{\mathrm{app}}$, e.g., $100^{\circ}, 120^{\circ}, 140^{\circ}$, and $160^{\circ}\left(R_{\mathrm{p}}=3.0 \mu \mathrm{m}, H=\right.$ $9.5 \mu \mathrm{m}$, and $f=0.3$ ).

that the collapse pressure increases significantly with the increase of $\theta_{\mathrm{adv}}^{\mathrm{app}}$ and $h / \mathrm{H}$. This is consistent with the experiments of Lee et al. ${ }^{14}$ by using structured sidewalls to enhance the stability of the liquid-air interface on superhydrophobic surfaces. Moreover, the experiments of Poetes et al. ${ }^{12}$ have shown that a sublevel roughness helps underwater superhydrophobic surfaces last longer under high hydrostatic pressure. Both phenomena observed by the above two experiments agree with our theoretical analysis. Therefore, we conclude that a hierarchical structure is important for the wetting stability on the submersed superhydrophobic surfaces under hydrostatic pressure from the point of view that it helps to strengthen the pinning of the three-phase contact line on the cavity sidewalls.

\section{CONCLUSIONS}

In this paper, we have investigated the wetting stability on the submersed superhydrophobic surfaces under hydrostatic pressure. A theoretical model for predicting the equilibrium states on the submersed substrates with the cylindrical cavities/ pillars is formulated. Comparison with the available experimental results shows that the depinning of the three-phase contact line may not induce the failure of the liquid-air interface, and a metastable state after depinning exists due to the increase of air pressure by the liquid pressurization. The advantage of the metastable state is that it can increase the liquid pressure that can be stood by superhydrophobic surfaces, and the disadvantage is that the depinned metastable state has higher free energy than the Wenzel state, and is not immune to external disturbances. In order to strengthen the stability of the depinned metastable state, the hierarchical structured surface is needed. It is shown that a two-level hierarchical surface with structured sidewalls is important for the stability of submersed superhydrophobicity in the way that it helps enhance the pinning of the liquid-air interface on the cavity sidewalls in the metastable state.

Although our formulation is based on the transition process from a CB to Wenzel state, a similar theoretical framework can be used to analyze the opposite transition direction, i.e., from a Wenzel to $\mathrm{CB}$ state. The present theory helps to better understand the wetting states and their stabilities on submersed superhydrophobic surfaces.

\section{AUTHOR INFORMATION}

\section{Corresponding Author}

*E-mail: hlduan@pku.edu.cn.

\section{Notes}

The authors declare no competing financial interest.

\section{ACKNOWLEDGMENTS}

Support by the following agencies and program is acknowledged: Major State Basic Research Development Program of China (Grant no. 2011CB013101). National Natural Science Foundation of China under Grants 10872003, 10932001 and 11172001; Program for the Author of National Excellent Doctoral Dissertation of China (grant no. 2007B2). Huiling Duan would like to acknowledge the Alexander von Humboldt $(\mathrm{AvH})$ foundation to support this work through project "Mechanics theory of materials with complex surfaces and its applications" in the frame of the AvH program for funding a research group linkage.

\section{REFERENCES}

(1) Cassie, A. B. D.; Baxter, S. Large Contact angles of plant and animal surfaces. Nature 1945, 155, 21-22.

(2) Wenzel, R. N. Resistance of solid surfaces to wetting by water. Ind. Eng. Chem. 1936, 28, 988-994.

(3) Quéré, D. Wetting and roughness. Annu. Rev. Mater. Res. 2008, $38,71-99$.

(4) Lafuma, A.; Quéré, D. Superhydrophobic states. Nat. Mater. 2003, 2, 457-460.

(5) Bormashenko, E.; Pogreb, R.; Whyman, G.; Erlich, G. Resonance Cassie-Wenzel wetting transition for horizontally vibrated drops deposited on a rough surface. Langmuir 2007, 23, 12217-12221.

(6) Kwon, H. M.; Paxson, A. T.; Varanasi, K. K.; Patankar, N. A. Rapid deceleration-driven wetting transition during pendant drop deposition on superhydrophobic surfaces. Phys. Rev. Lett. 2011, 106, 036102 .

(7) Bartolo, D.; Bouamrirene, F.; Verneuil, É; Buguin, A.; Silberzan, P.; Moulinet, S. Bouncing or sticky droplets: Impalement transitions on superhydrophobic micropatterned surfaces. EPL 2006, 74, 299305.

(8) Rothstein, J. P. Slip on superhydrophobic surfaces. Annu. Rev. Fluid Mech. 2010, 42, 89-109. 
(9) McHale, G.; Newton, M. I.; Shirtcliffe, N. J. Immersed superhydrophobic surfaces: Gas exchange, slip and drag reduction properties. Soft Matter 2010, 6, 714-719.

(10) Lei, L.; Li, H.; Shi, J.; Chen, Y. Diffraction patterns of a watersubmerged superhydrophobic grating under pressure. Langmuir 2010, 26, 3666-3669.

(11) Rathgen, H.; Mugele, F. Microscopic shape and contact angle measurement at a superhydrophobic surface. Faraday Discuss. 2010, $146,49-56$.

(12) Poetes, R; Holtzmann, K.; Franze, K.; Steiner, U. Metastable underwater superhydrophobicity. Phys. Rev. Lett. 2010, 105, 166104.

(13) Forsberg, P.; Nikolajeff, F.; Karlsson, M. Cassie-Wenzel and Wenzel-Cassie transitions on immersed superhydrophobic surfaces under hydrostatic pressure. Soft Matter 2011, 7, 104-109.

(14) Lobaton, E. J.; Salamon, T. R. Computation of constant mean curvature surfaces: Application to the gas-liquid interface of a pressurized fluid on a superhydrophobic surface. J. Colloid Interface Sci. 2007, 314, 184-198.

(15) Bhushan, B.; Jung, Y. C.; Koch, K. Micro-, nano- and hierarchical structures for superhydrophobicity, self-cleaning and low adhesion. Phil. Trans. R. Soc. London, Ser. A 2009, 367, 1631-1672.

(16) Liu, H. H.; Zhang, H. Y.; Li, W. Thermodynamic analysis on wetting behavior of hierarchical structured superhydrophobic surfaces. Langmuir 2011, 27, 6260-6267.

(17) Su, Y. W.; Ji, B. H.; Zhang, K.; Gao, H. J.; Huang, Y. G.; Hwang, K. Nature's design of hierarchical superhydrophobic surfaces of a water strider for low adhesion and low-energy dissipation. Langmuir 2010, 26, 18926-18937.

(18) Lee, C.; Kim, C. J. Maximizing the giant liquid slip on superhydrophobic microstructures by nanostructuring their sidewalls. Langmuir 2009, 25, 12812-12818.

(19) Patankar, N. A. Transition between superhydrophobic states on rough surfaces. Langmuir 2004, 20, 7097-7102.

(20) Marmur, A. Wetting on hydrophobic rough surfaces: To be heterogeneous or not to be. Langmuir 2003, 19, 8343-8348.

(21) Kusumaatmaja, H.; Blow, M. L.; Dupuis, A.; Yeomans, J. M. The collapse transition on superhydrophobic surfaces. EPL 2008, 81, 36003-1-6.

(22) Patankar, N. A. Hydrophobicity of surfaces with cavities: Making hydrophobic substrates from hydrophilic materials? J. Adhes. Sci. Technol. 2009, 23, 413-433.

(23) Liu, X. J.; Wang, X. L.; Liang, Y. M.; Bell, S. E. J.; Liu, W. M.; Zhou, F. Superoleophobicity under vacuum. Appl. Phys. Lett. 2011, 98, 194102.

(24) Bullard, J. W.; Garboczi, E. J. Capillary rise between planar surfaces. Phys. Rev. E 2009, 79, 011604.

(25) Zheng, Q. S.; Yu, Y.; Zhao, Z.-H. Effects of hydraulic pressure on the stability and transition of wetting modes of superhydrophobic surfaces. Langmuir 2005, 21, 12207-12212.

(26) Lee, D. G.; Kim., H. Y. The role of superhydrophobicity in the adhesion of a floating cylinder. J. Fluid Mech. 2009, 624, 23-32.

(27) Reyssat, M.; Quéré, D. Contact angle hysteresis generated by strong dilute defects. J. Phys. Chem. B 2009, 113, 3906-3909.

(28) Choi, W.; Tuteja, A.; Mabry, J. M.; Cohen, R. E.; McKinley, G. H. A modified Cassie-Baxter relationship to explain contact angle hysteresis and anisotropy on non-wetting textured surfaces. J. Colloid Interface Sci. 2009, 339, 208-216.

(29) Patankar, N. A. Hysteresis with regard to Cassie and Wenzel states on superhydrophobic surfaces. Langmuir 2010, 26, 7498-7503. 